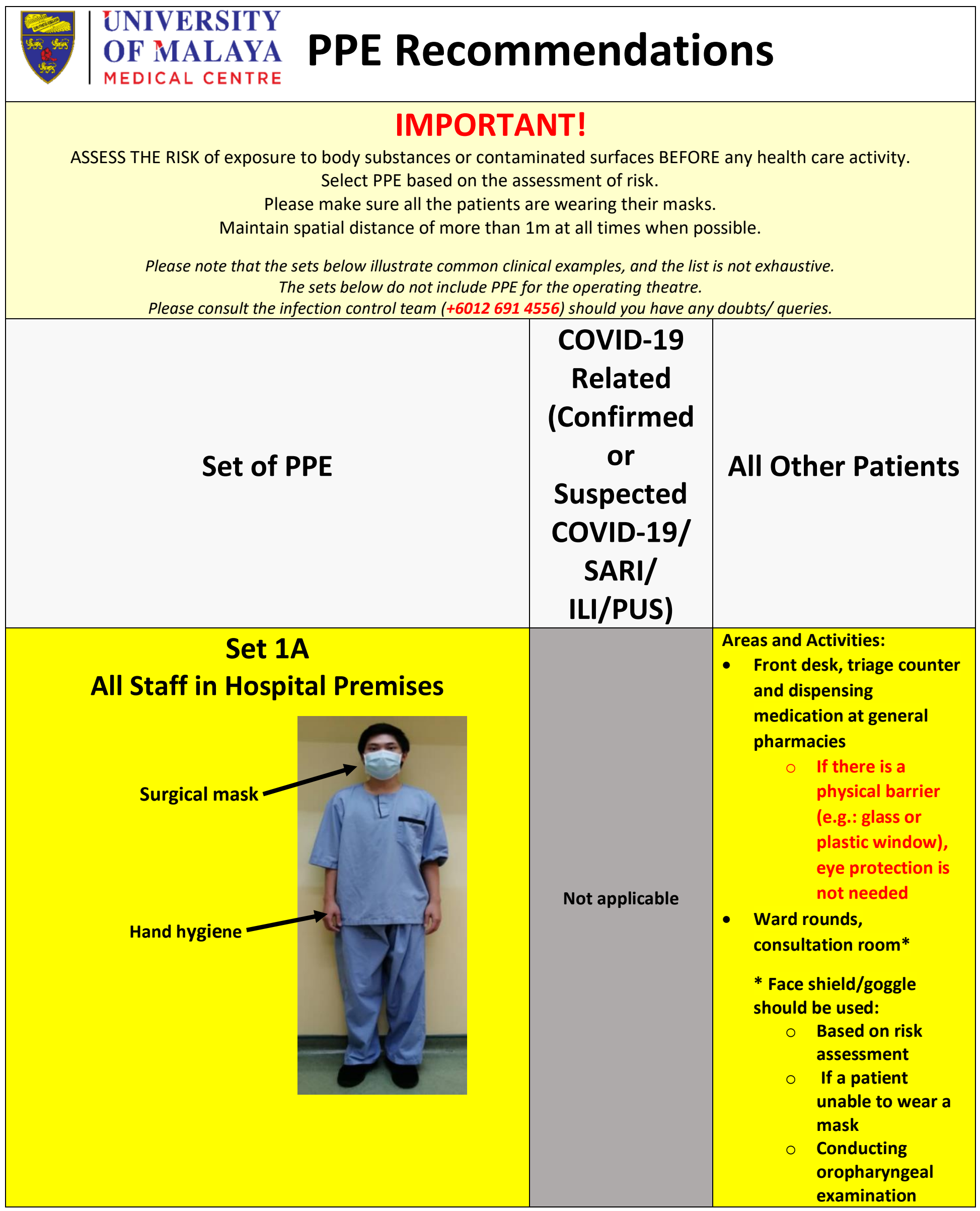




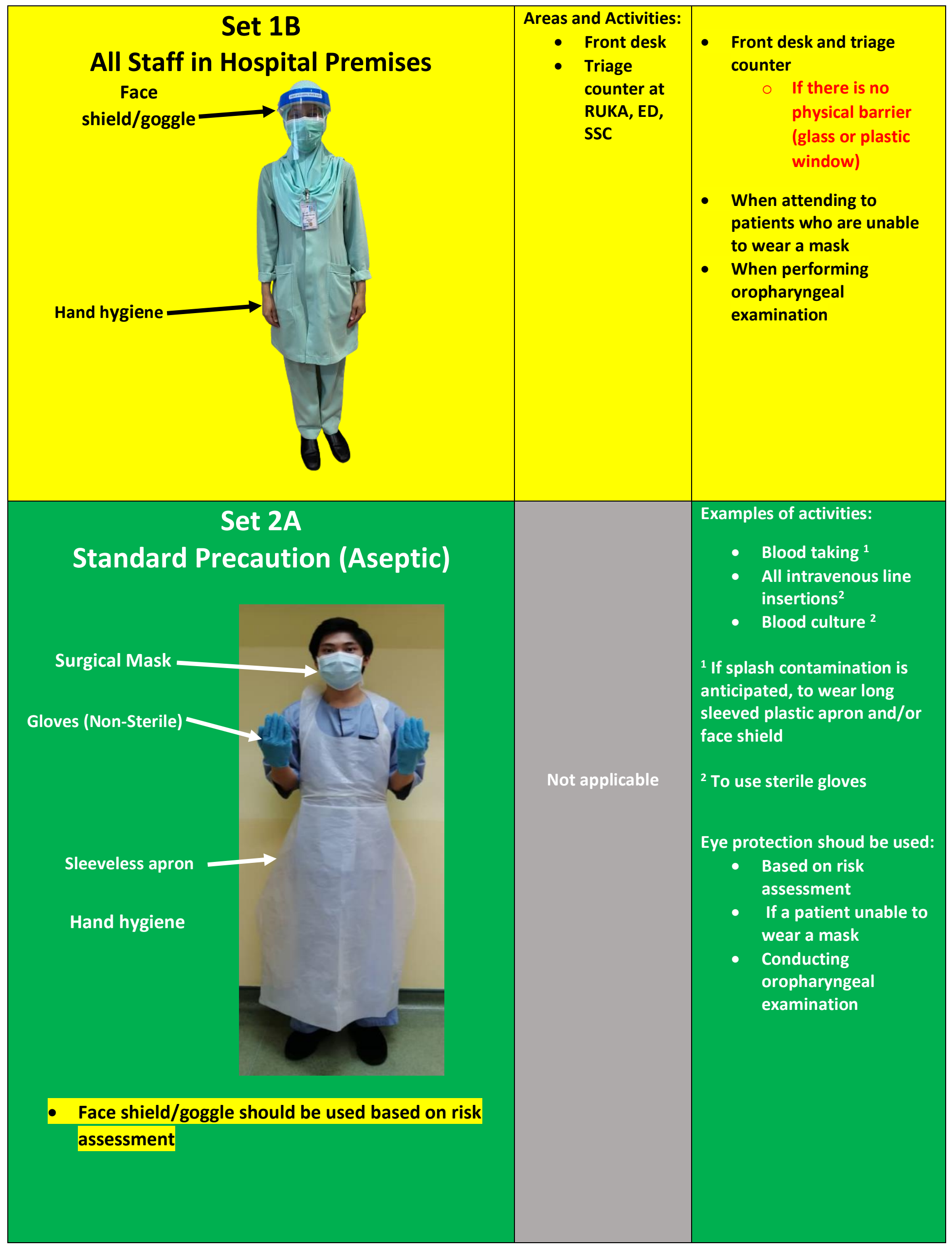




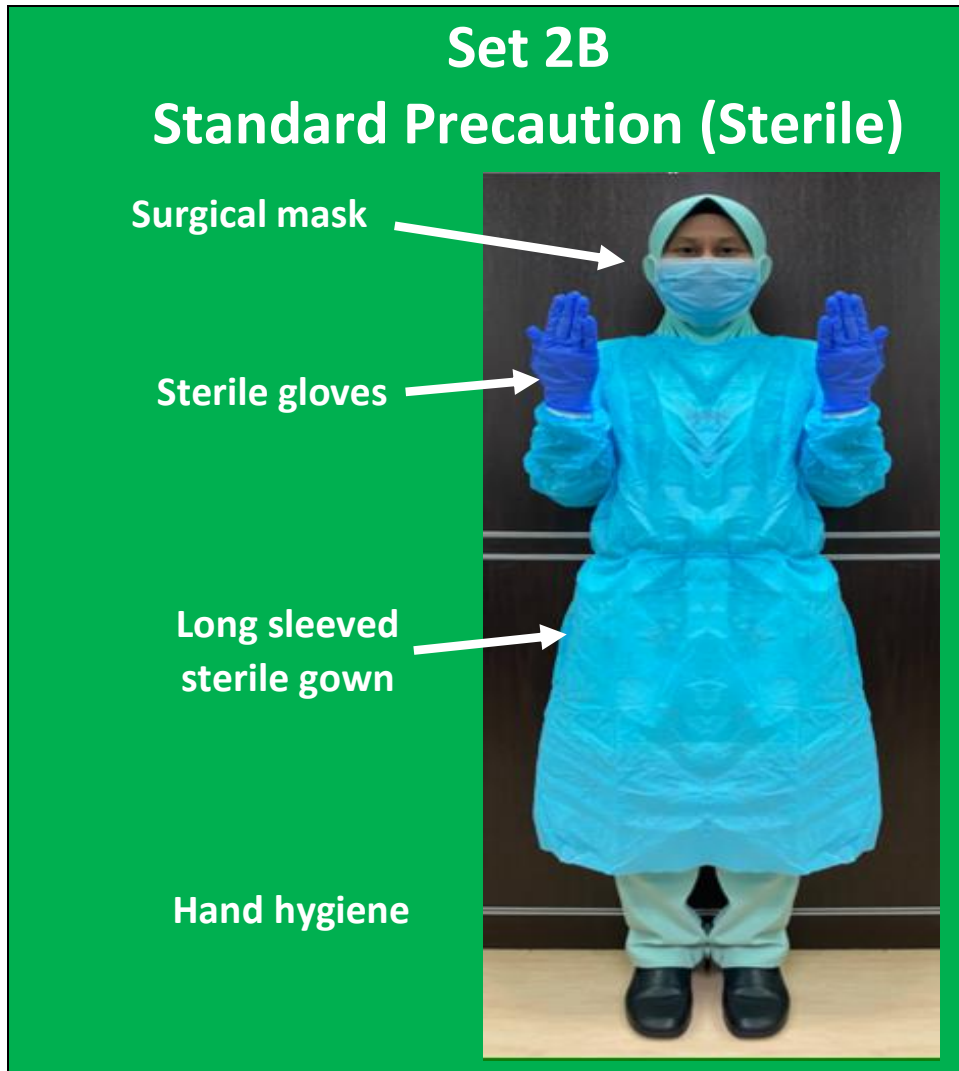

- Face shield/goggle should be used based on risk assessment

\section{Set 3}

\section{Standard \& Additional Precaution}

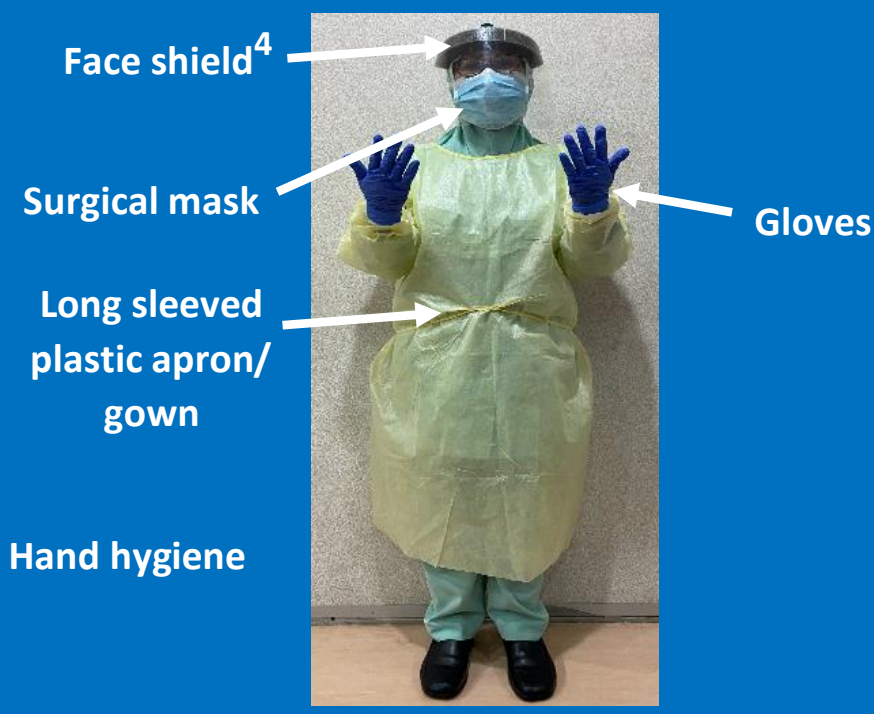

${ }^{4}$ For extended use:

- N95 mask and face shield

- To use gown together with plastic apron on top and to change plastic apron and gloves between patient
Examples of sterile procedures:

- Neuraxial anasthesia

- Peripheral nerve block

- Local anaesthesia

- Field blocks

- CVL and artline insertion/

- Removal

- Interventional radiology procedures e.g. thrombectomy, image-guided biopsies

- Angiogram/ Angioplasty

*Eye protection shoud be used:

- Based on risk assessment

- If a patient unable to wear a mask

- Conducting oropharyngeal examination

PPE use i.e.,

- $\quad \mathrm{N} 95$ mask/surgical face mask

- \pm eye/face protection

- \pm long sleeved apron/isolation gown

- \pm gloves

is based on the risk assessment done by the INDIVIDUAL healthcare worker, which is determined by the risk of contamination/splashes during the activity/procedure and the diagnosis of the patient)

- Asymptomatic

- Patient is able to wear a mask
Examples of activities:

- Care giving activities with risk of contamination or splash: i.e., Toileting care 


\section{High Risk $^{6}$}

Eye protection

(Face shield/ Goggles) ${ }^{4}$

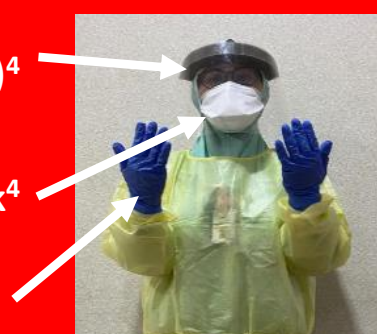

Long sleeved

plastic apron/

gown

\section{Hand hygiene}

\section{${ }^{4}$ For extended use:}

- $\quad$ N95 mask and face shield

- To use gown together with plastic apron on top and to change plastic apron and gloves between patient
Activities:

- Direct routine care of high risk patient

- Performing and assisting nasopharyngeal swab

6

High risk:

- Patient is coughing

- Patient requires oxygen support

- Patient unable to wear surgical mask

- Ventilated patient
Examples aerosol generating procedures/activities:

- Administration of medication via nebulization

- Obtaining nose and throat swabs

- Administration of pressurized humidified oxygen

- High flow nasal oxygenation

- Non-invasive ventilation e.g., BiPAP and CPAP

- Suctioning

- Sputum induction

- Chest physiotherapy

- Nasogastric tube insertion and feeding

- Clinical dysphagia examination

- this examination includes orofacial assessment and administration of food and/or fluids to evaluate swallowing ability

- Lower GI endoscopy

- Laser plume

- Active breathing circuit (Oncology)

- Intubation and extubation

- Manual ventilation

- Cardiopulmonary resuscitation

- Bronchoscopy

- Surgery with high speed devices used

- Post mortem procedures in which high-speed devices are used

- Dental procedures

- Front of neck airway procedures e.g. 


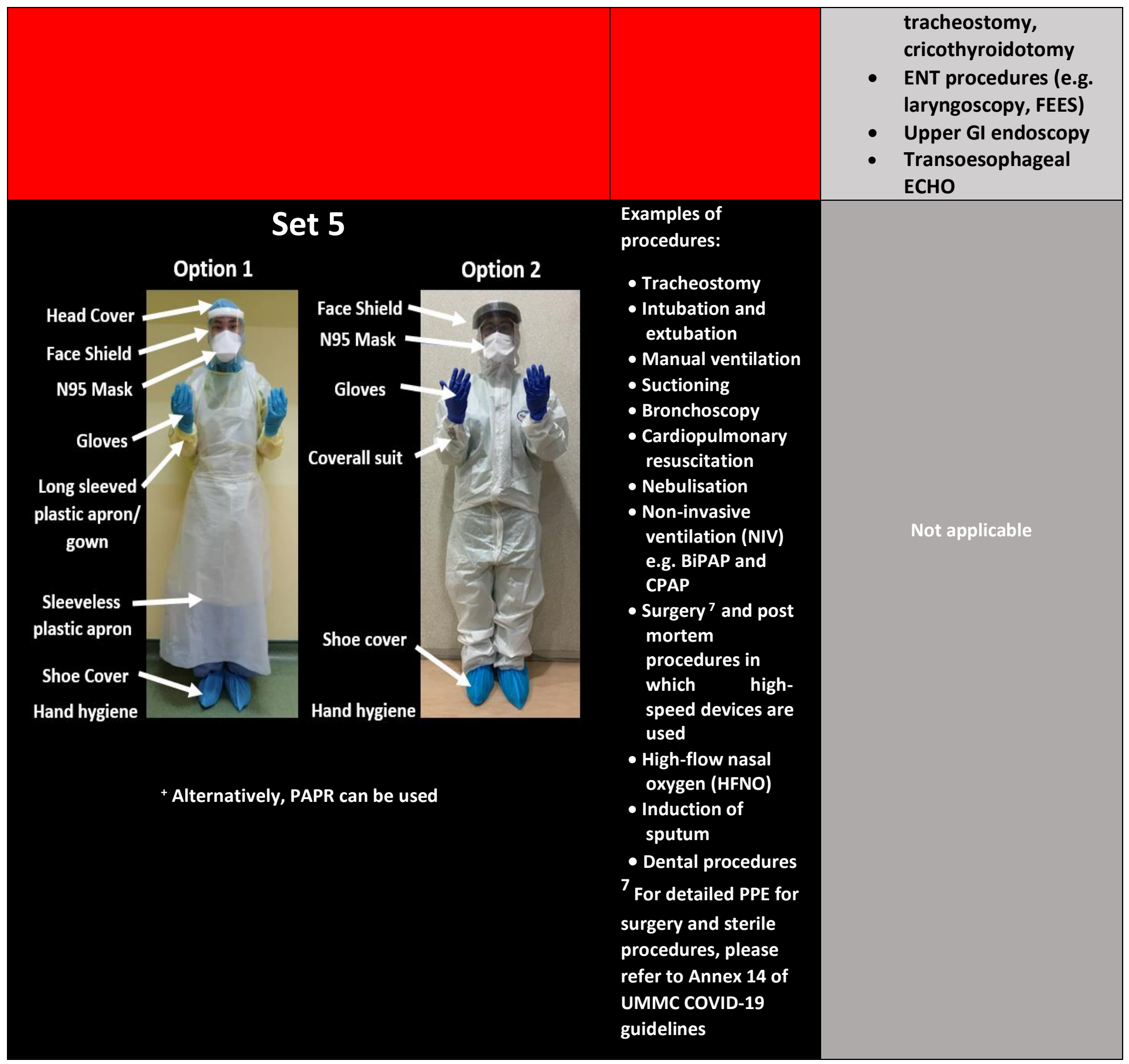

